\title{
A comparative analysis of diffusion- weighted imaging and ultrasound in thyroid nodules
}

\author{
Weidan Kong ${ }^{\dagger} \mathbb{D}$, Xiuhui Yue ${ }^{\dagger}$, Jiliang Ren and Xiaofeng Tao*
}

\begin{abstract}
Background: Diffusion-weighted imaging (DWI) and ultrasound are commonly used methods to examine thyroid nodules, but their comparative value is rarely studied. We evaluated the utility of DWI and ultrasound in differentiating benign and malignant thyroid nodules.

Methods: A total of 100 patients with 137 nodules who underwent both DWI and ultrasound before operation were enrolled. The T1 and T2 signal intensity ratio (SIR) of each thyroid nodule was calculated by measuring the mean signal intensity divided by that of paraspinal muscle. The apparent diffusion coefficient (ADC) value and the SIR of benign and malignant thyroid nodules were analyzed by two-sample independent $t$ tests. The sensitivity, specificity, and accuracy of DWI and ultrasound were compared with chi-square tests.

Results: There was no significant difference in the SIR between benign and malignant thyroid nodules. The ADC value was significantly different. At the threshold value was $1.12 \times 10^{-3} \mathrm{~mm}^{2} / \mathrm{s}$, the maximum area under the curve was 0.944 . The sensitivity, specificity, and accuracy were $84.9,92.2$, and $87.6 \%$ respectively. The corresponding values of ultrasound diagnosis were $90.1,80.4$, and $86.9 \%$.

Conclusions: Ultrasound has high sensitivity in differentiating benign and malignant thyroid nodules, and the ADC value has high specificity, but there is no statistical difference in sensitivity or specificity between the two modalities. DWI and ultrasound each have their own advantages in differentiating benign and malignant thyroid nodules.
\end{abstract}

Keywords: Thyroid nodules, Ultrasound, Diffusion-weighted imaging

\section{Background}

The incidence of thyroid malignant tumors has increased significantly in the past decades. The detection rate in healthy people ranges from 16 to $67 \%$ [1]. Most of the nodules tend to have a benign nature and differentiated thyroid cancer (mainly papillary and follicular carcinomas) accounts for the majority of cases, however, the malignancy has been reported in less than 5\% [2] of the nodules. If early detection and follow-up active treatment are carried out, the 10-year survival rate can be as high as $90 \%$ [3]. Ultrasound is the preferred method for thyroid tumor diagnosis. It has good diagnostic ability to

\footnotetext{
*Correspondence: cjr.taoxiaofeng@vip.163.com

'Weidan Kong and Xiuhui Yue contributed equally to this work.

Department of Radiology, Shanghai Ninth People's Hospital, Shanghai Jiao

Tong University School of Medicine, Shanghai, China
}

detect morphological manifestations, calcification, and cystic necrosis of thyroid lesions. However, previous studies have shown that diagnostic results are susceptible to the operator's influence [4]. Diffusion-weighted imaging (DWI) has become a popular modality for identifying thyroid nodules in recent years. Some researchers have suggested that the T2 signal intensity ratio (SIR) of thyroid nodules can be used as a quantitative parameter to differentiate benign from malignant nodules, which is of great significance for preoperative diagnosis [5]. The purpose of this study was to explore the best diagnostic threshold of DWI in differentiating benign and malignant thyroid nodules, and to compare the diagnostic value of DWI with ultrasound.

(C) The Author(s). 2019 Open Access This article is distributed under the terms of the Creative Commons Attribution 4.0 International License (http://creativecommons.org/licenses/by/4.0/), which permits unrestricted use, distribution, and reproduction in any medium, provided you give appropriate credit to the original author(s) and the source, provide a link to the Creative Commons license, and indicate if changes were made. The Creative Commons Public Domain Dedication waiver (http://creativecommons.org/publicdomain/zero/1.0/) applies to the data made available in this article, unless otherwise stated. 


\section{Methods}

\section{Patients}

The Institutional Review Board of Shanghai Ninth People's Hospital approved this retrospective study, and the requirement for informed consent was waived. The following criteria were adopted for patient selection: 1) primary thyroid tumor; 2) patients underwent both conventional DWI and ultrasound scan before treatment; 3) masses with short axis $\geq 10 \mathrm{~mm}$; 4) magnetic resonance (MR) images could be acquired and interpreted. Through a comprehensive search of our institutional medical report database from July 2017 to January 2019, we identified 100 patients (mean age, 49 years; range, 23-79 years) with 137 nodules (mean short axis, $18 \mathrm{~mm}$; range, $5-76 \mathrm{~mm}$ ). The final diagnoses based on histopathological results in 137 nodules, and the diagnostic results of DWI and ultrasound were compared.

\section{Image acquisition}

\section{DWI examination}

All MR imaging (MRI) examinations were performed on a $3.0 \mathrm{~T}$ scanner (Philips Ingenia $3.0 \mathrm{~T}$; Amsterdam, the Netherlands). The head and neck coils were placed over the thyroid surface. Patients were placed in a supine position with their neck, back, shoulders relaxed and instructed to breathe smoothly and avoid swallowing.

The MRI acquisition parameters were: T1WI turbo spin echo (TSE) (repetition time $[\mathrm{TR}]=450 \mathrm{~ms}$, echo time $[\mathrm{TE}]=20 \mathrm{~ms}$, thickness $=3 \mathrm{~mm}$, gap $=1 \mathrm{~mm}$, field of view $[\mathrm{FOV}]=240 \mathrm{~mm} \times 240 \mathrm{~mm}$, matrix $=300 \times 240$; T2WI DIXON-TSE $(\mathrm{TR}=2500 \mathrm{~ms}, \mathrm{TE}=100 \mathrm{~ms}$, thickness $=3 \mathrm{~mm}, \quad$ gap $=1 \mathrm{~mm}, \quad F O V=240 \mathrm{~mm} \times 240 \mathrm{~mm}$, matrix $=300 \times 240)$; DWI $\quad(\mathrm{TR}=400 \mathrm{~ms}, \quad \mathrm{TE}=70 \mathrm{~ms}$, thickness $=3 \mathrm{~mm}, \quad$ gap $=1 \mathrm{~mm}, \quad F O V=240 \mathrm{~mm} \times 240$ $\mathrm{mm}$, matrix $=220 \times 180$ ). The $\mathrm{b}$ values were 0 and 1000 $\mathrm{s} / \mathrm{mm}^{2}$.

\section{Ultrasound examination}

All ultrasound examinations were performed on a ultrasound scanner (Toshiba-Aplio 400; Tokyo, Japan). The probe frequency was 5-12 MHz. During examination, the patient was in a supine position, with the head slightly backward to fully expose the neck. The probe was placed over the thyroid region. The nodule boundary, echo, blood flow, calcification, and peripheral lymph node enlargement were observed.

\section{Image analysis}

Apparent diffusion coefficient (ADC) measurements were made by two radiologists with 3 and 7 years of experience in head and neck imaging, who were blinded to the clinical information and diagnosis. DWI image analysis was performed on a Philips post-processing workstation to measure the average ADC value of each nodule (b value $0,1000 \mathrm{~s} / \mathrm{mm}^{2}$ ) and the SIRs on T1WI and T2WI. For the ADC value, a region of interest (ROI) of the same size on different sections was selected for multi-point measurement, and the average value was obtained. The ROIs were selected while avoiding cystic degeneration, hemorrhage, and necrosis as much as possible. The SIR was calculated for each sequence as a ratio of signal intensity of the thyroid nodule to that of the paraspinal muscle.

Ultrasound examination was performed by a radiologist with 7 years of experience in head and neck imaging, who was blinded to clinical information and diagnosis. The Thyroid Imaging Reporting AND Date System (TIRADS) classification method was used in the ultrasound diagnosis report, which stipulated that grade 1-3a was benign and grade $3 \mathrm{~b}-5$ was malignant. Finally, each nodule was given a grade and qualitative diagnosis.

\section{Statistical analysis}

SPSS 20.0 software (IBM Corp., Armonk, NY) was used for statistical analysis. Differences in ADC, T1SIR, and T2SIR in patients with benign and malignant thyroid nodules were evaluated by independent sample $t$ tests. Difference were considered statistically significant when $P<0.05$. The ADC thresholds for differentiating benign and malignant thyroid nodules were obtained from receiver operating characteristic (ROC) curves. Finally, the diagnostic values of ultrasound and DWI were compared by paired chi-square tests.

\section{Results \\ Pathology}

A total of 137 thyroid nodules were found in $100 \mathrm{pa}$ tients (21 male and 79 female), including 79 in the left lobe, 51 in the right lobe, and 7 in the isthmus. There were 86 malignant nodules (82 papillary, 2 follicular, and 2 lymphoma) and 51 benign nodules (31 nodular goiters, 11 thyroid adenomas, and 9 Hashimoto's thyroiditis).

\section{ADC and SIR}

The average ADC value of benign thyroid nodules $\left(1.36 \pm 0.17 \times 10^{-3} \mathrm{~mm}^{2} / \mathrm{s}\right)$ was higher than that of malignant thyroid nodules $\left(0.89 \pm 0.21 \times 10^{-3} \mathrm{~mm}^{2} / \mathrm{s}\right)$, and the difference was statistically significant $(P<0.05$, Fig. 1$)$. There were no significant differences in T1SIR or T2SIR between benign and malignant thyroid nodules $(P>$ 0.05). According to ROC curve analysis, the best ADC threshold for differentiating benign and malignant thyroid nodules was $1.12 \times 10^{-3} \mathrm{~mm}^{2} / \mathrm{s}$. The maximum area under the corresponding curve was 0.944 , and the sensitivity and specificity were 84.9 and $92.2 \%$, respectively (Fig. 2). The ADC values and T1 and T2 SIRs of benign and malignant thyroid nodules are detailed in Table 1. 


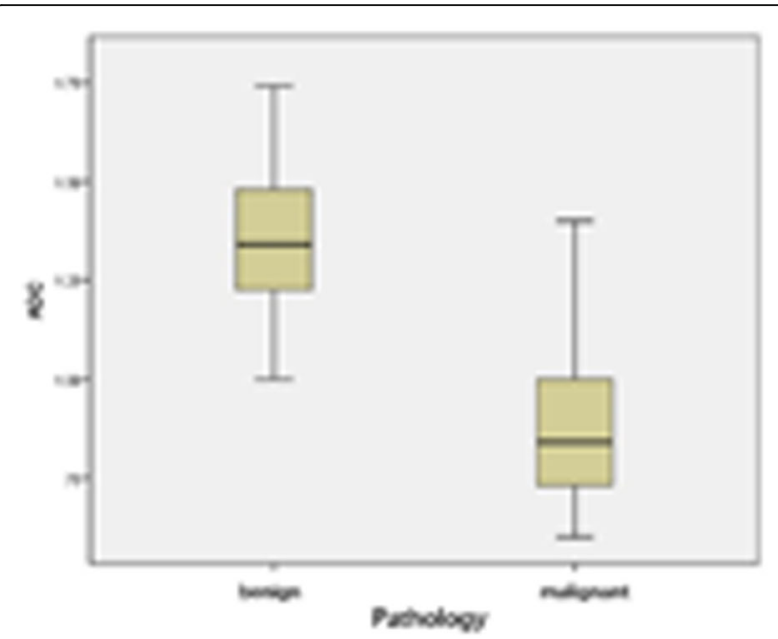

Fig. 1 Box plot shows apparent diffusion coefficient (ADC) values for malignant and benign thyroid nodules. The bottom, middle, and top box lines indicate the 25th percentile, median, and 75th percentile, respectively. The error bars show the smallest and largest values in 1.5-box lengths of the 25th and 75th percentiles

\section{Diagnostic results of DWI and ultrasonography}

There were 82 papillary thyroid carcinomas (Fig. 3) and 3 follicular thyroid carcinomas. Most showed high or slightly high signals on DWI, and the signals were relatively uniform. Two lymphomas showed nodular high signals on DWI, and the internal signals were relatively uniform. Thirty-one nodular goiters (Fig. 4) and nine Hashimoto's thyroiditis cases showed slightly higher nodular signal intensity, as did 11 thyroid adenomas.

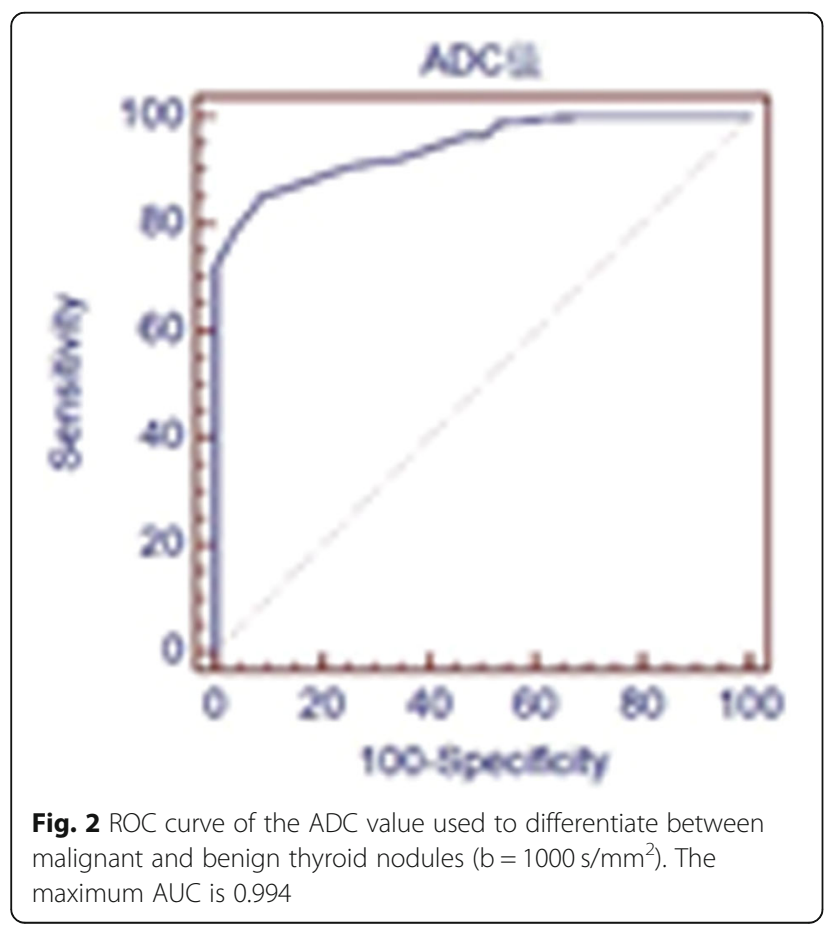

In 137 nodules, 77 were malignant $(73$ consistent with pathological findings) and 60 were benign (47 consistent with pathological findings). The sensitivity, specificity, and accuracy of MRI differential diagnosis were 84.9, 92.2 , and $87.6 \%$ respectively.

The TI-RADS classification method was used in the ultrasound diagnosis report (grade 1-3a was benign and grade $3 \mathrm{~b}-5$ was malignant). Among 137 nodules, 88 were malignant (78 consistent with pathological findings) and 49 were benign ( 41 consistent with pathological findings). The sensitivity, specificity, and accuracy of ultrasound diagnosis were 90.1, 80.4, and $86.9 \%$ respectively. Finally, the qualitative diagnostic results of MRI and ultrasound were compared by chi-square test. The utility of these modalities for diagnosing thyroid nodules is compared in detail in Table 2.

\section{Discussion}

In this study, 15 cases of malignant nodules were associated with Hashimoto's thyroiditis and 2 cases with nodular goiter. Noda et al. [5] reported a statistical difference between benign and malignant thyroid nodules on T2SIR $(P<0.001)$, and the authors suggested that dense fibrous tissue in papillary carcinomas led to a decrease in T2SIR. In this study, we found no significant difference between T1 and T2SIR $(P>0.05)$, and the SIRs of benign and malignant nodules were smaller than those reported previously, which may be related to the type of machine or the type of samples. At present, there are few studies on the SIR, so it is premature to use T2SIR as a stable index for thyroid nodule identification.

Nodules were characterized by differential lobes or irregular margins, microcalcification, or mixed calcification based on the TI-RADS grading method [6]. Cheng et al. [7] reported that the blood flow characteristics of nodules could be used as an important reference index for differentiating benign and malignant nodules. However, there were some overlaps between benign and malignant nodules on sonograms, and previous studies suggested [5, 8] that the TI-RADS classification is still controversial. Malignant nodules have blurred boundaries, solid hypoechoic interiors, gravel-like calcification, and mixed internal blood flow, while lymphoma nodules have abundant internal blood flow signals. Ota et al. [9] reported that an enhanced posterior echo of nodules is an important differentiating sign of lymphoma compared to other malignant thyroid tumors; benign nodules have clear boundaries, low-signal and isoechoic interiors, and may be accompanied by coarse calcium. Most of the lesions had complete capsules, with rare blood flow signals in the nodules. Circular blood flow signals could be seen around the nodules, while blood flow was abundant in Hashimoto's thyroiditis nodules. In this study, the sensitivity of ultrasound diagnosis of nodules was high 
Table 1 Comparison of ADC values and signal intensity ratios of T1 and T2 (SIR) in benign and malignant thyroid nodules

\begin{tabular}{llll}
\hline MR sequence & Benign nodule $(n=51)$ & Malignant nodule $(n=86)$ & $P$ value \\
\hline ADC & $1.36 \pm 0.17(1.31 \sim 1.40)$ & $0.89 \pm 0.21(0.84 \sim 0.93)$ & $<0.05$ \\
T1SIR & $1.77 \pm 0.72(1.57 \sim 1.97)$ & $1.81 \pm 0.57(1.69 \sim 1.94)$ & 0.42 \\
T2SIR & $1.80 \pm 0.49(1.66 \sim 1.94)$ & $1.64 \pm 0.41(0.84 \sim 0.93)$ & 0.07 \\
\hline
\end{tabular}

Note: The data in the table are expressed by mean (+standard deviation) and the confidence interval range in brackets is $95 \%$. $P<0.05$ indicates that there is statistical difference

(90.1\%), but the specificity was not (80.4\%). Compared with MRI, ultrasound is more sensitive for detecting micro-lesions and identifying micro-calcification. Almost all calcifications were detected. However, due to inherent limitations of ultrasound technology, the exploration of deep cervical tissue and the evaluation of peripheral lymph node metastasis are inferior to MRI.

The use of DWI has been increasingly studied for the differential diagnosis of benign and malignant thyroid nodules. The ADC value is related to the free movement of water molecules but is also affected by microcirculation perfusion. Wang et al. [10] concluded that the ADC value is an independent measurement factor in the differential diagnosis of benign and malignant thyroid nodules in multi-parameter DWI studies. We found that the $\mathrm{ADC}$ values of benign thyroid nodules were significantly higher than those of malignant thyroid nodules, which was consistent with previous studies [3, 10, 11]. Wu et al. [11] reported that DWI can provide ample information about tissue microstructure and physiological processes. The existence of organelles and biomacromolecules in tissues limits the free movement of extracellular water molecules. Conversely, Schueller-Weidekamm et al. [12] suggested that ADC values in malignant nodules were higher than those in benign nodules. The authors of that study proposed that increased follicular production in malignant nodules increases ADC values. In this study, the ADC value of malignant nodules $\left(0.89 \pm 0.21 \times 10^{-3} \mathrm{~mm}^{2} / \mathrm{s}\right)$ was lower than that of benign nodules $\left(1.36 \pm 0.17 \times 10^{-3} \mathrm{~mm}^{2} / \mathrm{s}\right)$. The main reason was that the number and density of malignant nodules were more than that of benign nodules, which resulted in decreased intercellular space. At the same time, the malignant nodules were mainly papillary carcinomas, which have increased mitosis and a higher nuclearcytoplasmic ratio, corresponding to limited intracellular water molecule activity. Fibrosis also hampers the diffusion of water molecules to varying degrees, which is consistent with Cheng et al. [7]. Liu et al. [13] . suggested that irregular nodule margins are a strong predictor of
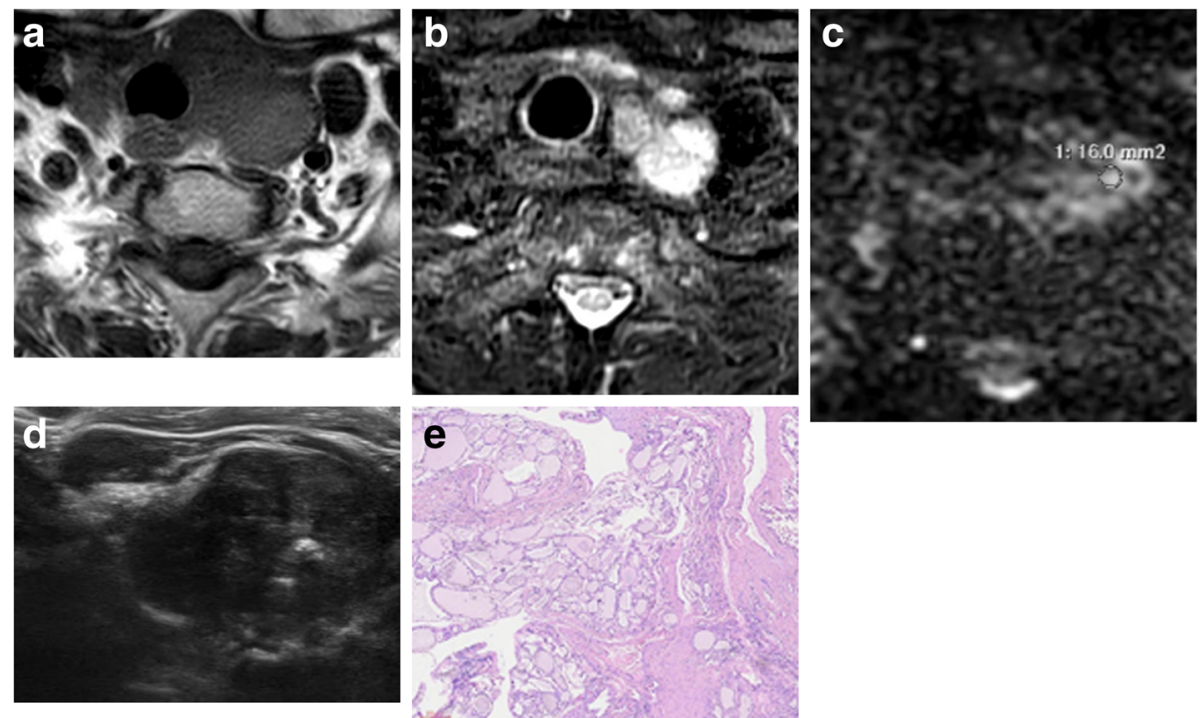

Fig. 3 A 45-year-old man with papillary carcinoma of the left lobe of the thyroid (white arrow). a: axial T1Wl showed low signal with multiple nodules in the left lobe, $\mathbf{b}$ : axial T2WI fat suppression on the same level showed high signal with unclear boundary, $\mathbf{c}$ : ADC map showed obvious high signal on the same level, and the ADC value is $1.0 \times 10^{-3} \mathrm{~mm}^{2} / \mathrm{s}$, $\mathbf{d}$ : Ultrasound transverse section showed hypoechoic nodule in left thyroid lobe, irregular shape, unclear boundary, uneven echo, and multiple hyperechoic and hypoechoic points. TI-RADS:4c. e: HE staining (10*40)

showed irregular and branching papillary in nodules, with packed arranged glassy cells with atypical nuclear, intranuclear inclusions and nuclear grooves were also observed, fibrous strips (pseudocapsule) were seen at the edge, and neoplastic cell nests infiltrated into the capsule 

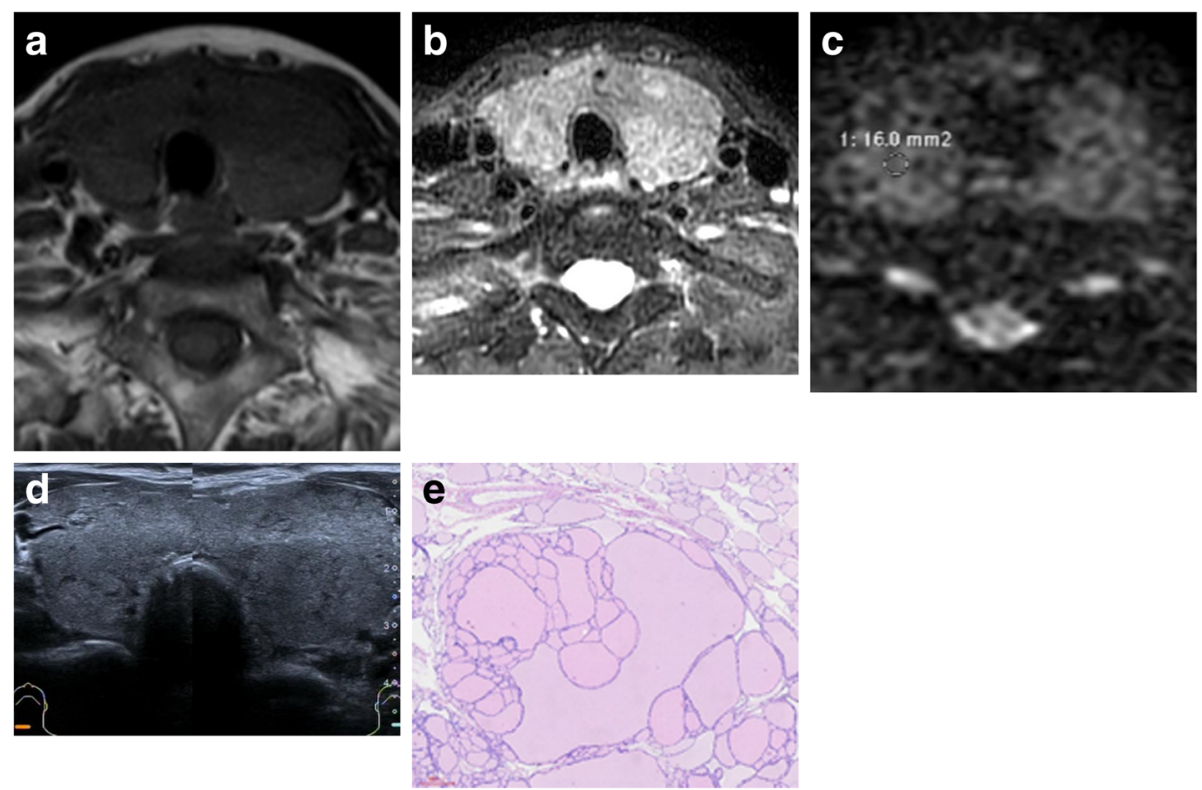

Fig. 4 A 63-year-old woman with a bilateral nodular goiter of the thyroid (white arrow). a: axial T1WI showed bilateral lobe enlargement with low-signal shadows, $\mathbf{b}$ : axial T2WI fat suppression on the same level showed high signal with unclear boundary, c: ADC map showed obvious high signal on the same level, and the ADC value is $1.8 \times 10^{-3} \mathrm{~mm}^{2} / \mathrm{s}$, d: Ultrasound transverse section showed that bilateral thyroid lobes were plump, isthmus was thicker, surface was smooth, echo was medium, thickening and uneven distribution, TI-RADS:3a. e:HE staining (10*40) showed that the tightly packed follicles were filled with colloid, some endocytosins of different sizes absorbed vacuolesv vacuoliation, and microvessels decreased between follicles

malignant tendency. Malignant nodules on DWI usually have high signal intensity. Quantitative ADC values obtained from post-processing can objectively and qualitatively evaluate nodules. Using $1.12 \times 10^{-3} \mathrm{~mm}^{2} / \mathrm{s}$ as the threshold, we found higher specificity of DWI diagnosis (92.2\%) and lower sensitivity (84.9\%). Compared with ultrasound, the detection rate of lymph node metastasis was higher. According to Abdel Razek et al. [14], DWI combined with ADC maps can help identify metastatic non-necrotic lymph nodes and provide microscopic information about lymph node status. Recently, Wang et al. [15] concluded that a high b value $\left(2000 \mathrm{~s} / \mathrm{mm}^{2}\right)$ has high accuracy for the differential diagnosis of benign and malignant thyroid micronodules.

Our results should be interpreted in the context of several limitations. Firstly, it was a retrospective study with relatively small number of patients, the preponderance of malignant nodules in the sample selection will

Table 2 Comparison of sensitivity and specificity of MR and ultrasound in differential diagnosis of benign and malignant thyroid nodules (\%)

\begin{tabular}{llll}
\hline Inspection mode & Sensitivity & Specificity & Accuracy \\
\hline MRI (ADC) & $84.9 \%(73 / 86)$ & $92.2 \%(47 / 51)$ & $87.6 \%$ \\
Ultrasound & $90.1 \%(18 / 86)$ & $80.4 \%(41 / 51)$ & $86.9 \%$ \\
$X^{2}$ & 0.44 & 0.82 & 31.2 \\
$P$ & $>0.05$ & $>0.05$ & $<0.05$ \\
\hline
\end{tabular}

inevitably affect some quantitative parameters of DWI. Secondly, the malignant group only included papillary, follicular, and lymphoma subtypes. Thirdly, we adopted b values of 0 and $1000 \mathrm{~s} / \mathrm{mm}^{2}$ for DWI. A b values of $800 \mathrm{~s} / \mathrm{mm}^{2}$ was more commonly used in previous thyroid studies. Finally, ultrasound examination results were based on the opinion of one diagnostic physician, which is subjective. Therefore, we will continue to expand the sample sizes and types, perform comparative analysis with ultrasound, and conduct an in-depth comparative study of differences between groups.

\section{Conclusions}

The threshold of the ADC value for differentiating benign and malignant thyroid nodules was $1.12 \times 10^{-3}$ $\mathrm{mm}^{2} / \mathrm{s}$. There was no statistical difference in sensitivity or specificity between ultrasound and DWI. Ultrasound is superior to DWI for detecting calcification, while MRI is more useful for diagnosing lymph node metastasis. Both modalities play important roles in thyroid nodule diagnosis.

\footnotetext{
Abbreviations

ADC: Apparent diffusion coefficient; AUC: Area under the ROC curve; DWI: Diffusion-weighted imaging; FOV: Field of view; MRI: Magnetic resonance imaging; ROC: Receiver operating characteristic; ROl: Region of interest; SIR: Signal intensity ratio; TE: Echo time; TI-RADS: Thyroid imaging reporting and date system; TR: Repetition time; TSE: turbo spin echo
} 


\section{Acknowledgements}

Not applicable.

\section{Authors' contributions}

$X T$ and WK conceived and designed the study. WK, XY, and JR collected the data. WK and XY analyzed the data and wrote the paper. All authors read and approved the final manuscript for publication.

\section{Authors' information}

All the authors are from the Department of Radiology, Shanghai Ninth People's Hospital, Shanghai Jiao Tong University School of Medicine. XT is the director of the Radiology department, and WK is a postgraduate of Medical College of Shanghai Jiaotong University.

\section{Funding}

This work was supported by funds from the National Scientific Foundation of China $(91859202,81771901)$

\section{Availability of data and materials}

The dataset supporting the conclusions of this article is available upon request to the corresponding author.

\section{Ethics approval and consent to participate}

The study was approved by Institutional Review Board of Shanghai Ninth People's Hospital. Individual consent was waived due to the retrospective study design.

\section{Consent for publication}

Not applicable.

\section{Competing interests}

The authors declare that they have no competing interests.

Received: 24 July 2019 Accepted: 20 September 2019

Published online: 21 November 2019

\section{References}

1. Yoon JH, Lee HS, Kim EK, Moon HJ, Kwak JY. Malignancy risk stratification of thyroid nodules: comparison between the thyroid imaging reporting and data system and the 2014 American Thyroid Association management guidelines. Radiology. 2016;278(3):917-24.

2. Hegedus L, Bonnema SJ, Bennedbaek FN. Management of simple nodular goiter: current status and future perspectives [J]. Endocr Rev. 2003;24(1): 102-32

3. Khizer AT, Raza S, Slehria AU. Diffusion-weighted MR imaging and ADC mapping in differentiating benign from malignant thyroid nodules. J Coll Physicians Surg Pak. 2015;25(11):785-8.

4. Ko SY, Lee HS, Eun-Kyung KKim EK, Kwak JY. Application of the thyroid imaging reporting and data system in thyroid ultrasonography interpretation by less experienced physicians. Ultrasonography. 2014;33(1): 49-57.

5. Noda Y, Kanematsu M, Goshima S, Kondo H, Watanabe H, Kawada H, et al MRI of the thyroid for differential diagnosis of benign thyroid nodules and papillary carcinomas. AJR Am J Roentgenol. 2015;204(3):332-5.

6. Kwak JY, Han KH, Yoon JH, Moon HJ, Son EJ, Park SH, et al. Thyroid imaging reporting and data system for US features of nodules: a step in establishing better stratification of cancer risk. Radiology. 2011;260(3):892-9.

7. Cheng PW, Chou HW, Wang CT, Lo WC, Liao LJ. Evaluation and development of a real-time predictive model for ultrasound investigation of malignant thyroid nodules. Eur Arch Otorhinoaryngol. 2014;271(5):1199-206.

8. Sasaki M, Sumi M, Kaneko K, Ishimaru K, Takahashi H, Nakamura T. Multiparametric MR imaging for differentiating between benign and malignant thyroid nodules: initial experience in 23 patients. J Magn Reason Imaging. 2013;38(1):64-71.

9. Ota H, Ito YF, Matsuzuka F, Kuma S, Fukata S, Morita S, et al. Usefulness of ultrasonography for diagnosis of malignant lymphoma of the thyroid. Thyroid. 2006;16(10):983-7.

10. Wang H, Wei R, Liu W, Chen Y, Song B. Diagnostic efficacy of multiple MR parameters in differentiating benign vs. malignant thyroid nodules. BMC Med Imaging. 2018;18(1):50.
11. Wu Y, Yue X, Shen W, Du Y, Yuan Y, Tao X, et al. Diagnostic value of diffusion-weighted MR imaging in thyroid disease: application in differentiating benign from malignant disease. BMC Med Imaging. 2013; 13(1):23.

12. Schueller-Weidekamm C, Kaserer K, Schueller G, Scheuba C, Ringl H, Weber $M$, et al. Can quantitative diffusion-weighted MR imaging differentiate benign and malignant cold thyroid nodules? Initial results in 25 patients. AJNR Am J Neuroradiol. 2009;30(2):417-22.13.

13. Liu J, Zheng D, Li Q, Tang X, Luo Z, Yuan Z, et al. A predictive model of thyroid malignancy using clinical, biochemical and sonographic parameters for patients in a multi-center setting. BMC Endocr Disord. 2018;18(1):17.

14. Abdel Razek AA, Soliman NY, Elkhamary S, Alsharaway MK, Tawfik A. Role of diffusion-weighted MR imaging in cervical lymphadenopathy. Eur Radiol. 2006;16(7):1468-77.

15. Wang Q, Guo Y, Zhang J, Ning H, Zhang X, Lu Y, et al. Diagnostic value of high b-value $\left(2000 \mathrm{~s} / \mathrm{mm}^{2}\right)$ DWI for thyroid micronodules. Medicine (Baltimore). 2019;98(10):e 14298.

\section{Publisher's Note}

Springer Nature remains neutral with regard to jurisdictional claims in published maps and institutional affiliations.

\section{Ready to submit your research? Choose BMC and benefit from:}

- fast, convenient online submission

- thorough peer review by experienced researchers in your field

- rapid publication on acceptance

- support for research data, including large and complex data types

- gold Open Access which fosters wider collaboration and increased citations

- maximum visibility for your research: over $100 \mathrm{M}$ website views per year

At $\mathrm{BMC}$, research is always in progress.

Learn more biomedcentral.com/submissions 